\title{
SPECTRUM REDUCING EXTENSION FOR ONE OPERATOR ON A BANACH SPACE
}

\author{
C. J. READ
}

\begin{abstract}
In this paper we show that, given an operator $T$ on a Banach space $X$, there is an extension $Y$ of $X$ such that $T$ extends in a natural way to an operator $T^{\sim}$ on $Y$, and the spectrum of $T^{\sim}$ is the approximate point spectrum of $T$. This answers a question posed by Bollobas, and contributes to a theory investigated by Shilov, Arens, Bollobás, etc. The unusual transfinite construction is similar to that which we used earlier to find an inverse producing extension for a commutative unital Banach algebra which eliminates the residual spectrum of one element. We also give a counterexample, consisting of a Banach algebra $L$ containing elements $g_{1}$ and $g_{2}$ such that in no extension $L^{\prime}$ of $L$ are the residual spectra of $g_{1}$ and $g_{2}$ eliminated simultaneously.
\end{abstract}

1. Introduction. Our result is clearly best possible in the sense that if an element of the spectrum is removed in some extension, then that element cannot have come from the approximate point spectrum.

The argument used to construct the extension $Y$ of $X$ involves transfinite induction on the set $\Omega$ of countable ordinals introduced for a similar purpose in our earlier paper [1]. We have since used similar methods again in [7] to deal with the special case when $X$ is a Hilbert space; in this case, $Y$ can be a Hilbert space also.

\section{Preliminary definitions.}

DEFINITION 2.1. Let $\Omega$ denote the set of all countable ordinals. We shall tend to use the symbols $\alpha, \beta$, and $\gamma$ for elements of $\Omega$.

DEFINITION 2.2. Given the operator $T$ on a Banach space $X$, let $\sigma_{\mathrm{ap}}(T)$ be the approximate point spectrum of $T$; that is, let

$$
\sigma_{\text {ap }}(T)=\{\lambda \in \mathrm{C}: \inf \{\|(\lambda I-T) x\|:\|x\|=1\}=0\} .
$$

$\sigma_{\mathrm{ap}}(T)$ is well known to be compact and nonempty.

DEFINITION 2.3. Let us choose, once and for all, a collection of neighborhoods $\left(U_{i}\right)_{i=1}^{\infty}$ of $\sigma_{e}(T)$ with the following properties.

(1) For each $i \in \mathbf{N}, U_{i} \supset \bar{U}_{i+1}$ (where $\bar{U}$ denotes the closure of $U$ ).

(2) Every component of $U_{i}$ intersects $\sigma_{\mathrm{ap}}(T)$.

(3) $\bigcap_{i=1}^{\infty} U_{i}=\sigma_{\mathrm{ap}}(T)$.

DEFINITION 2.4. Given a Banach space $Z$, we define certain larger Banach spaces as follows.

Received by the editors June 2, 1985 and, in revised form, June 11, 1987.

1980 Mathematics Subject Classification (1985 Revision). Primary 47A20.

Key words and phrases. Extension, inverse producing, spectrum reducing, functional calculus, transfinite induction. 
(1) Let $l_{\infty}(Z)$ be the collection of all bounded sequences $\left(z_{i}\right)_{i=1}^{\infty} \subset Z$, with the supremum norm

$$
\left\|\left(z_{i}\right)_{i=1}^{\infty}\right\|=\sup _{i \in \mathbf{N}}\left\|z_{i}\right\|_{Z}
$$

Let $j_{Z}: Z \rightarrow l_{\infty}(Z)$ be the isometric embedding of $Z$ in $l_{\infty}(Z)$ which sends $z \in Z$ to the constant sequence $\left(z_{i}\right)_{i=1}^{\infty}$ such that each $z_{i}=z$.

(2) Let $c_{0}(Z)$ be the closed subspace of $l_{\infty}(Z)$ consisting of those sequences in $Z$ which norm converge to zero.

(3) Let $Z^{+}$denote the quotient space $l_{\infty}(Z) / c_{0}(Z)$, and let $\pi_{Z}: Z \rightarrow Z^{+}$be the composition of the inclusion map $j_{Z}$ with the quotient map $\rho_{Z}: l_{\infty}(Z) \rightarrow$ $l_{\infty}(Z) / c_{0}(Z)$.

NOTE 2.5. $\pi_{Z}: Z \rightarrow Z^{+}$is an isometric embedding.

DEFINITION 2.6. Given a Banach space $Z$, let $Z^{+}$and $\pi_{Z}: Z \rightarrow Z^{+}$be as in Definition 2.4. Then for each operator $S \in L(Z)$ we define an extension $S^{+} \in$ $L\left(Z^{+}\right)$as follows:

$$
S^{+}\left(\left(z_{i}\right)_{i=1}^{\infty}+c_{0}(Z)\right)=\left(S z_{i}\right)_{i=1}^{\infty}+c_{0}(Z) .
$$

NoTE 2.7. Indeed, the map $S \rightarrow S^{+}$defines an isometric embedding of $L(Z)$ in $L\left(Z^{+}\right)$.

DEFINITION 2.8. We now proceed to define a collection of Banach spaces $X_{\alpha}$, one for each ordinal $\alpha \in \Omega$. The $X_{\alpha}$ 's are directed by linking maps $\tau_{\alpha \beta}: X_{\alpha} \rightarrow X_{\beta}$ $(\alpha<\beta \in \Omega)$ which are isometric embeddings. They must also satisfy the following conditions.

(1) $X_{1}=X$, our original Banach space.

(2) For each $\alpha, X_{\alpha+1}$ is the Banach space $X_{\alpha}^{+}$, and $\tau_{\alpha, \alpha+1}$ is the map $\pi_{X_{\alpha}}: X_{\alpha} \rightarrow$ $X_{\alpha}^{+}$. For each $\gamma<\alpha, \tau_{\gamma, \alpha+1}$ must then be $\tau_{\alpha, \alpha+1} \circ \tau_{\gamma, \alpha}$.

(3) For each limit ordinal $\beta$, let $\hat{X}_{\beta}$ denote the direct limit of the sets $\left\{X_{\alpha}: \alpha<\right.$ $\beta$, directed by the linking maps $\tau . \hat{X}_{\beta}$ inherits a normed vector space structure from the $X_{\alpha}$ 's, $\alpha<\beta$. The space $X_{\beta}$ is the completion of $\hat{X}_{\beta}$, and each linking $\operatorname{map} \tau_{\alpha \beta}(\alpha<\beta)$ is the composition of the direct limit of the maps $\tau_{\alpha \gamma}(\alpha<\gamma<\beta)$ with the embedding of $\hat{X}_{\beta}$ in the completion $X_{\beta}$.

By transfinite induction, there is just one collection $\left(X_{\alpha}\right)_{\alpha \in \Omega}$ satisfying these conditions. Since we need to have a natural way of extending our operator $T$ from $X$ to the larger spaces $X_{\alpha}$, we must have one more inductive definition in this section.

DEFinition 2.9. Given the operator $T$ we proceed to define extensions $T_{\alpha}$ of $T$ to the larger spaces $X_{\alpha}$ as follows, beginning with $T_{1}=T$.

(1) For each $\alpha, T_{\alpha+1}=\left(T_{\alpha}\right)^{+}$.

(2) For each limit ordinal $\beta$, let $\hat{T}_{\beta}$ denote the direct limit of the maps $T_{\alpha}, \alpha<\beta$. $T_{\beta}$ is the unique continuous extension of $\hat{T}_{\beta} \in L\left(\hat{X}_{\beta}\right)$ to the completion $X_{\beta}$ of $\hat{X}_{\beta}$.

Once again, there is just one collection of extensions $T_{\alpha}$, given the original operator $T$. The map $T \rightarrow T_{\alpha}$ defines an isometric embedding of $L(X)$ in $L\left(X_{\alpha}\right)$.

3. Definition of an admissible sequence. We are now ready to define a new collection of Banach spaces, one of which will be the extension of $X$ which we require. 
DEFinition 3.1. For each $\alpha \in \Omega, n \in \mathbf{N}$, let $V_{\alpha}^{(n)}$ be the vectorspace of all bounded analytic functions $U_{n} \rightarrow X_{\alpha}$. Let $\|\cdot\|_{\alpha}^{(n)}$ denote the supremum norm on $V_{\alpha}^{(n)}$

$$
\|f\|_{\alpha}^{(n)}=\sup _{z \in U_{n}}\|f(z)\|_{X_{\alpha}}
$$

There is a natural embedding of $X_{\alpha}$ in $V_{\alpha}^{(n)}$ as the constant functions, and we shall denote this embedding by $\psi_{\alpha}^{(n)}$. If no confusion is possible about the values of $\alpha$ and $n$, we will just write $\psi$ for our embedding.

We wish to impose a more complicated norm on $V_{\alpha}^{(n)}$, determined by a sequence of constants $\left(\varepsilon_{i}\right)_{i=1}^{n}$ which we shall choose. We shall always assume that each $\varepsilon_{i}$ is a positive real number less than 1 , and the sequence $\left(\varepsilon_{i}\right)_{i=1}^{n}$ is monotonic decreasing. Given such a sequence, we define a norm in the following way.

DEFINITION 3.2. Given a sequence $\varepsilon=\left(\varepsilon_{i}\right)_{i=1}^{n}$ we define a norm on $V_{\alpha}^{(n)}$ as follows: for all $f \in V_{\alpha}^{(n)}$,

$$
\begin{aligned}
& \|f\|_{\alpha}^{(\varepsilon)}=\inf \left\{\|c\|_{X_{\alpha}}+\sum_{i=1}^{n}\left\|f_{i}\right\|_{\alpha}^{(i)}: c \in X_{\alpha}, f_{i} \in V_{\alpha}^{(i)}\right. \\
& \left.\qquad+\sum_{i=1}^{n} \varepsilon_{i} f_{i}(z)=f(z) \text { for all } z \in U_{n}\right\} .
\end{aligned}
$$

We note that for all $f \in V_{\alpha}^{(n)}$,

$$
\|f\|_{\alpha}^{(n)} \leq\|f\|_{\alpha}^{(\varepsilon)} \leq \varepsilon_{n}^{-1} \cdot\|f\|_{\alpha}^{(n)},
$$

so $\|\cdot\|_{\alpha}^{(\varepsilon)}$ is a genuine norm on $V_{\alpha}^{(n)}$. The embedding $\psi$ is obviously an isometric embedding of $X_{\alpha}$ in $\left(V_{\alpha}^{(n)},\|\cdot\|_{\alpha}^{(\varepsilon)}\right)$ as the constant functions. Not at all obvious, though, is the fact that we can embed $X_{\alpha}$ in a similar way in a certain quotient space of $V_{\alpha}^{(n)}$, defined below.

Recall that $T$ is our given operator on $X$ whose spectrum we wish to reduce. For each $\alpha \in \Omega$, let $T_{\alpha}$ be the extension of $T$ to the space $X_{\alpha}$ defined in $\S 2.7$.

Definition 3.3. (1) For each $\alpha \in \Omega, n \in \mathbf{N}$, let $Z_{\alpha}^{(n)}$ denote the $\|\cdot\|_{\alpha}^{(n)}$ closed subspace of $V_{\alpha}^{(n)}$ generated by those analytic functions $f \in V_{\alpha}^{(n)}$ which satisfy

$$
\begin{aligned}
f(z) & =z g(z)-T_{\alpha} \circ g(z) \\
& =\left(z I-T_{\alpha}\right) \circ g(z)
\end{aligned}
$$

for all $z \in U_{n}$, where $g$ is another element of $V_{\alpha}^{(n)}$.

(2) Let $Y_{\alpha}^{(n)}$ be the quotient space $V_{\alpha}^{(n)} / Z_{\alpha}^{(n)}$, and let $\Psi_{\alpha}^{(n)}$ be the map $X_{\alpha} \rightarrow$ $Y_{\alpha}^{(n)}$ obtained by composing the embedding

$$
\psi: X_{\alpha} \rightarrow V_{\alpha}^{(n)}
$$

with the quotient map $V_{\alpha}^{(n)} \rightarrow V_{\alpha}^{(n)} / Z_{\alpha}^{(n)}$. We shall write

$$
\left(Y_{\alpha}^{(n)},\|\cdot\|_{\alpha}^{(n)}\right), \quad\left(Y_{\alpha}^{(n)},\|\cdot\|_{\alpha}^{(\varepsilon)}\right)
$$

for the normed quotient spaces obtained from $\left(V_{\alpha}^{(n)},\|\cdot\|_{\alpha}^{(n)}\right),\left(V_{\alpha}^{(n)},\|\cdot\|_{\alpha}^{(\varepsilon)}\right)$ respectively. 
We are crucially interested in the map $\Psi$; we claim that for a suitable choice of the sequence $\varepsilon$ the map $\Psi: X_{\alpha} \rightarrow\left(Y_{\alpha}^{(n)},\|\cdot\|_{\alpha}^{(\varepsilon)}\right)$ is an isometric embedding of $X_{\alpha}$ in $Y_{\alpha}^{(n)}$. Hence, we make the following definition.

DEFINITION 3.4. The sequence $\left(\varepsilon_{i}\right)_{i=1}^{n}$ is said to be admissible for the ordinal $\alpha \in \Omega$ if the map $\Psi: X_{\alpha} \rightarrow\left(Y_{\alpha}^{(n)},\|\cdot\|_{\alpha}^{(\varepsilon)}\right)$ is an isometry.

Our claim is as as follows.

THEOREM 3.5. There is a decreasing sequence $\left(\varepsilon_{i}\right)_{i=1}^{\infty}$ of strictly positive reals such that for every $n \in \mathbf{N}$ and every $\alpha \in \Omega$, the sequence $\left(\varepsilon_{i}\right)_{i=1}^{n}$ is admissible for $\alpha$.

The proof of this consists of several stages.

4. Existence of admissible sequences. The general outline of the proof that there is a sequence as described in Theorem 3.5 is as follows.

First we observe that if $\left(\varepsilon_{i}\right)_{i=1}^{n}$ is admissible for $\alpha$ then so is any $\left(\varepsilon_{i}^{\prime}\right)_{i=1}^{n}$ with each $\varepsilon_{i}^{\prime} \leq \varepsilon_{i}$. Next, we prove that there is an $\varepsilon_{1}>0$ which is admissible for every $\alpha \in \Omega$. Then we assume as an induction hypothesis that there is a sequence $\left(\varepsilon_{i}\right)_{i=1}^{n}$ admissible for all $\alpha \in \Omega$, and prove the following.

For every $0<\delta<1$ there is an $\varepsilon_{n+1}>0$ such that the sequence $\delta \varepsilon_{1}, \delta \varepsilon_{2}, \delta \varepsilon_{3}, \ldots$, $\delta \varepsilon_{n}, \varepsilon_{n+1}$ is admissible for every $\alpha \in \Omega$.

However, this completes the proof, since we can then choose sequences

$$
\begin{aligned}
& \varepsilon_{1} \\
& \delta_{1} \varepsilon_{1}, \varepsilon_{2} \\
& \delta_{2} \delta_{1} \varepsilon_{1}, \delta_{2} \varepsilon_{2}, \varepsilon_{3} \\
& \delta_{3} \delta_{2} \delta_{1} \varepsilon_{1}, \delta_{3} \delta_{2} \varepsilon_{2}, \delta_{3} \varepsilon_{3}, \varepsilon_{4}
\end{aligned}
$$

and so on, where each sequence is admissible for all $\alpha \in \Omega$, and $\prod_{i=1}^{\infty} \delta_{i}>\frac{1}{2}$. But then the sequence $\left(\frac{1}{2} \varepsilon_{i}\right)_{i=1}^{\infty}$ satisfies our requirements.

Let us then make our preliminary observation concerning admissible sequences.

LEMMA 4.1. If $\left(\varepsilon_{i}\right)_{i=1}^{n}$ is admissible for $\alpha$ and $\left(\varepsilon_{i}^{\prime}\right)_{i=1}^{n}$ satisfies $0<\varepsilon_{i}^{\prime}<\varepsilon_{i}$ for each $i$, then $\left(\varepsilon_{i}^{\prime}\right)_{i=1}^{n}$ is admissible for $\alpha$.

PROOF. The map $\psi: X_{\alpha} \rightarrow\left(V_{\alpha}^{(n)},\|\cdot\|_{\alpha}^{(\varepsilon)}\right)$ is an isometry, and the quotient $\operatorname{map} V_{\alpha}^{(n)} \rightarrow Y_{\alpha}^{(n)}$ is of norm less than or equal to 1 , so certainly $\|\Psi(x)\|_{\alpha}^{(\varepsilon)} \leq\|x\|$ for all $x \in X_{\alpha}$, and similarly $\|\Psi(x)\|_{\alpha}^{\left(\varepsilon^{\prime}\right)} \leq\|x\|$ for all $x \in X_{\alpha}$. The question of admissibility depends on whether the reverse inequality holds, that is

$$
\begin{aligned}
\|x\| & \leq\|\Psi(x)\|_{\alpha}^{(\varepsilon)} \\
& =\inf \left\{\|f\|_{\alpha}^{(\varepsilon)}: f \in V_{\alpha}^{(n)}, \text { and for some } g \in V_{\alpha}^{(n)}, f+(z I-T) \circ g=x\right\} .
\end{aligned}
$$

However, the norm $\|\cdot\|_{\alpha}^{(\varepsilon)}$ certainly increases with decreasing $\varepsilon$, so if this inequality holds for some values $\left(\varepsilon_{i}\right)_{i=1}^{n}$ it also holds with all smaller values $\left(\varepsilon_{i}^{\prime}\right)_{i=1}^{n}$.

Our next task is to prove that there is an $\varepsilon_{1}>0$ such that $\varepsilon_{1}$ is admissible for all $\alpha \in \Omega$. 
Fix an $\alpha \in \Omega$. Let us prove that there is an $\varepsilon_{1}>0$ such that $\varepsilon_{1}$ is admissible for $\alpha$. We require the following lemma.

LEMMA 4.2. The approximate point spectrum of $T_{\alpha}$ is the same as the approximate point spectrum of $T$.

PROOF. We shall in fact show more than this; in fact, for each $\alpha$,

$$
\inf \left\{\left\|\left(\lambda I-T_{\alpha}\right) x\right\|: x \in X_{\alpha},\|x\|=1\right\}
$$

is equal to

$$
\inf \{\|(\lambda I-T) x\|: x \in X,\|x\|=1\} .
$$

Since $\lambda$ is in the approximate point spectrum if and only if the appropriate infimum is zero, this clearly proves the result. Moreover, $T_{\alpha}$ is an extension of $T$ to a larger space $X_{\alpha}$, so it is certain that expression (4.2.1) is less than or equal to (4.2.2). Conversely, we claim that for all $x \in X_{\alpha}, \lambda \in \mathbf{C}$ such that $\|x\|=1$, we have

$$
\left\|\left(\lambda I-T_{\alpha}\right) x\right\| \geq \inf \{\|(\lambda I-T) y\|: y \in X,\|y\|=1\} .
$$

The statement is clearly true for $\alpha=1$, when $X_{\alpha}=X$ and $T_{\alpha}=T$. Proceeding therefore by transfinite induction, let us assume the result is true for a particular ordinal $\alpha$. If

$$
\mathbf{x} \in X_{\alpha+1}=l_{\infty}\left(X_{\alpha}\right) / c_{0}\left(X_{\alpha}\right),
$$

let us say $\mathbf{x}$ is in the equivalence class of the bounded sequence

$$
\left(x_{i}\right)_{i=1}^{\infty} \in X_{\alpha} .
$$

Then

$$
\left\|\left(\lambda I-T_{\alpha+1}\right) \mathbf{x}\right\|=\left\|\left[\left(\left(\lambda I-T_{\alpha}\right) x_{i}\right)_{i=1}^{\infty}\right]\right\|_{l_{\infty}\left(X_{\alpha}\right) / c_{0}\left(X_{\alpha}\right)}
$$

(where [.] denotes the equivalence class of the sequence)

$$
\begin{aligned}
& =\limsup \left\|\left(\lambda I-T_{\alpha}\right) x_{i}\right\|_{X_{\alpha}} \\
& \geq \limsup \left\|x_{i}\right\|_{X_{\alpha}} \cdot \inf _{y \in X_{\alpha}} \frac{\left\|\left(\lambda I-T_{\alpha}\right) y\right\|}{\|y\|} \\
& =\limsup \left\|x_{i}\right\|_{X_{\alpha}} \cdot \inf \left\{\left\|\left(\lambda I-T_{\alpha}\right) y\right\|: y \in X_{\alpha},\|y\|=1\right\} \\
& =\|\mathbf{x}\|_{X_{\alpha+1}} \cdot \inf \left\{\left\|\left(\lambda I-T_{\alpha}\right) y\right\|: y \in X_{\alpha},\|y\|=1\right\} \\
& =\|\mathbf{x}\| \cdot \inf \{\|(\lambda I-T) w\|: w \in X,\|w\|=1\}
\end{aligned}
$$

by the induction hypothesis.

So if our claim is true for the ordinal $\alpha$ it is also true for $\alpha+1$. Moreover, an inequality such as (4.2.3) is preserved under direct limits and completions, so it is true for all $\alpha \in \Omega$.

Returning to the main problem of showing that, given $\alpha$,

$$
\Psi: X_{\alpha} \rightarrow\left(Y_{\alpha}^{(1)},\|\cdot\|_{\alpha}^{\left(\varepsilon_{1}\right)}\right)
$$

is an isometry for some $\varepsilon_{1}>0$, we note that since

$$
\|\Psi(x)\|=\inf \left\{\|f\|_{\alpha}^{\left(\varepsilon_{1}\right)}: f \in V_{\alpha}^{(1)} \text {, and for some } g \in V_{\alpha}^{(1)}, f+\left(z I-T_{\alpha}\right) \circ g=x\right\},
$$

it is certainly necessary that there should be no nonzero constant $x \in X_{\alpha}$ which can be represented as

$$
\left(z I-T_{\alpha}\right) \circ g(z)
$$


for all $z$ in $U_{1}$, and some bounded analytic $g$. We know, however, that each $U_{n}$ contains the approximate point spectrum of $T_{\alpha}$ (for it is equal to the approximate point spectrum of $T$ ), so it is sufficient to prove the following lemma.

LEMMA 4.4. Let $B$ be a Banach space, $T \in L(B)$, and let $U$ and $V$ be open sets in $\mathbf{C}$ such that

(1) $U$ contains the approximate point spectrum of $T$,

(2) $V$ contains $U$, and

(3) every component of $V$ intersects $U$.

Suppose we have

$$
f(\lambda)=(\lambda I-T) \circ g(\lambda)
$$

for all $\lambda \in U$, where $f$ and $g$ are analytic functions $f: V \rightarrow B$ and $g: U \rightarrow B$. Then there is an analytic extension $\hat{g}: V \rightarrow B$ of $g$.

Note: The proof that follows is very similar to that of Lemma 3 of [1].

PROOF OF LEMMA 4.4. We can find an open set $W \subset \mathbf{C}$ such that $V=U \cup W$ and $\bar{W} \cap \sigma_{\text {ap }}(T)=\varnothing$. Each component of $W$ will intersect $U$, and our problem is to extend the analytic germs of $g$ from $U$ to all of $W$.

Since

$$
\bar{W} \cap \sigma_{\mathrm{ap}}(T)=\varnothing
$$

we claim there is an $\eta>0$ such that $\lambda \in W, x \in B$ implies

$$
\|(\lambda I-T) x\| \geq \eta\|x\| .
$$

For if not, there are sequences $\left(\lambda_{n}\right)_{n=1}^{\infty} \subset W$ and $\left(x_{n}\right)_{n=1}^{\infty} \subset B$ such that $\left\|x_{n}\right\|=1$ but $\left\|\left(\lambda_{n} I-T\right) x_{n}\right\| \rightarrow 0$. Then $\left\{\lambda_{n}: n \in \mathbf{N}\right\}$ must be bounded, so, taking a subsequence as necessary, we may assume that $\lambda_{n} \rightarrow \lambda \in \bar{W}$. Then

$$
\begin{aligned}
\left\|(\lambda I-T) x_{n}\right\| & \leq\left\|\left(\lambda-\lambda_{n}\right) x_{n}\right\|+\left\|\left(\lambda_{n} I-T\right) x_{n}\right\| \\
& =\left|\lambda-\lambda_{n}\right|+\left\|\left(\lambda_{n} I-T\right) x_{n}\right\| \\
& \rightarrow 0 \quad \text { as } n \rightarrow \infty .
\end{aligned}
$$

Thus $\lambda \in \sigma_{\mathrm{ap}}(T)$, so $\lambda \in \bar{W} \cap \sigma_{\mathrm{ap}}(T)$, contradicting the fact that this set is empty.

Let us choose an $\eta>0$ such that (4.4.2) is satisfied. Now,

$$
f(\lambda)=(\lambda I-T) \circ g(\lambda)
$$

which implies that, for each $n=1,2,3, \ldots$,

$$
f^{(n)}(\lambda)=(\lambda I-T) \circ g^{(n)}(\lambda)+n g^{(n-1)}(\lambda)
$$

where $h^{(r)}$ denotes the $r$ th derivative of a function $h$. It follows that, if we have an analytic germ of $g$ at some point $\lambda_{0} \in W$, then

$$
\left\|g^{(n)}\left(\lambda_{0}\right)\right\| \leq \sum_{r=0}^{n}\left\|f^{(r)}\left(\lambda_{0}\right)\right\| \cdot \frac{n !}{r !} \cdot\left(\frac{1}{\eta}\right)^{n-r+1}
$$

for all $n \in \mathrm{N}$. So if the power series for $f$ at $\lambda_{0}$ has radius of convergence $\delta>0$, then the power series for $g$ at $\lambda_{0}$ has radius of convergence greater than or equal to $\eta \wedge \delta$.

Therefore the radius of convergence is bounded away from zero on any compact set in $W$; hence it must be possible to extend $g$ throughout $W$, as required. Thus Lemma 4.4 is proved. 
COROLlaRY 4.5. Suppose for some ordinal $\alpha \in \Omega, x \in X_{\alpha}$, and analytic function $g: U_{n} \rightarrow X_{\alpha}$ we have, for all $z \in U_{n}$,

$$
\left(z I-T_{\alpha}\right) \circ g(z)=x \text {. }
$$

Then $x=g=0$.

ProOF. By Lemma 4.2, $\sigma_{\mathrm{ap}}\left(T_{\alpha}\right)=\sigma_{\mathrm{ap}}(T) \subset U_{n}$. Therefore we can apply Lemma 4.4 with $B=X_{\alpha}, T=T_{\alpha}, U=U_{n}$ and $V=\mathrm{C}$. Then the constant function $f(z)=x$ is indeed analytic throughout $\mathbf{C}$, and $f(\lambda)=\left(\lambda I-T_{\alpha}\right) \circ g(\lambda)$ for all $\lambda \in U_{n}$. So $g$ extends throughout $\mathbf{C}$ to a bounded entire function, which must be constant. Therefore $x=g=0$.

Let us now prove that there is an $\varepsilon_{1}>0$ which is admissible for a given $\alpha \in \Omega$.

LEMMA 4.6. For each $\alpha \in \Omega$, there is an $\varepsilon>0$ such that $\varepsilon$ is admissible for $\alpha$.

PROOF. It is required (in view of (4.1.1)) that we show that there is an $\varepsilon>0$ such that for all $x \in X_{\alpha}$,

$$
\|\Psi(x)\|_{\alpha}^{(\varepsilon)} \geq\|x\|
$$

where the norm on $Y_{\alpha}^{(1)}$ of the equivalence class of the function $x$ is

$$
\begin{gathered}
\inf \left\{\|f\|_{\alpha}^{(\varepsilon)}: f \in V_{\alpha}^{(1)}, f(z)+\left(z I-T_{\alpha}\right) \circ g(z)=x \text { for all } z \in U_{1}, \text { some } g \in V_{\alpha}^{(1)}\right\} \\
=\inf \left\{\|c\|_{X_{\alpha}}+\left\|f_{1}\right\|_{\alpha}^{(1)}: c \in X_{\alpha}, f_{1} \in V_{\alpha}^{(1)}, \text { and for some } g \in V_{\alpha}^{(1)}\right. \\
\text { we have } \left.c+\varepsilon_{1} f_{1}(z)+(z I-T) \circ g(z)=x \text { for all } z \in U_{1}\right\} .
\end{gathered}
$$

If this is less than $\|x\|$ for a particular value of $\varepsilon$ then there are $c, x \in X_{\alpha}$,

$$
\begin{gathered}
c+\varepsilon_{1} f_{1}+(z I-T) \circ g=x \text { on } U_{1}, \\
\|c\|+\left\|f_{1}\right\|_{\alpha}^{(1)}<\|x\| .
\end{gathered}
$$

So writing $d=x-c$, we have

$$
\begin{gathered}
\varepsilon_{1} f_{1}+(z I-T) \circ g=d, \\
\left\|f_{1}\right\|_{\alpha}^{(1)}<\|x\|-\|c\| \leq\|d\| .
\end{gathered}
$$

Suppose there is no $\varepsilon>0$ such that $\varepsilon$ is admissible for $\alpha$. Then there are elements $\left(d_{i}\right)_{i=1}^{\infty} \in X_{\alpha}$, without loss of generality $\left\|d_{i}\right\|=1$ for all $i$, such that for all $i$,

$$
\begin{gathered}
d_{i}=2^{-i} f_{i}+(z I-T) \circ g_{i}, \\
\left\|f_{i}\right\|_{\alpha}^{(1)}<1 .
\end{gathered}
$$

Now let $W_{1}$ be any open set such that $W_{1} \subset U_{1}, \bar{W}_{1} \cap \sigma_{\mathrm{ap}}(T)=\varnothing$, and $\partial W_{1} \supset \partial U_{1}$. Then as in the proof of Lemma 4.4, there is an $\eta>0$ such that

$$
\|(\lambda I-T) x\| \geq \eta\|x\|
$$

for all $x \in X_{\alpha}, \lambda \in W_{1}$; hence, for all $\lambda \in W_{1}$,

$$
\left\|g_{i}(\lambda)\right\| \leq \frac{1}{\eta}\left\|(z I-T) \circ g_{i}(\lambda)\right\|=\frac{1}{\eta}\left\|d_{i}-2^{-i} f_{i}(\lambda)\right\| \leq \frac{1}{\eta}\left(1+2^{-i}\right) .
$$

So the $g_{i}$ 's are uniformly bounded on $W_{1}$, hence, since $\partial W_{1} \supset \partial U_{1}$, the $g_{i}$ 's are uniformly bounded throughout $U_{1}$ by the maximum modulus principle, and indeed 
are uniformly differentiable on compact sets in $U_{1}$. Therefore we may define an analytic function

$$
\begin{aligned}
G & : U_{1} \rightarrow l_{\infty}\left(X_{\alpha}\right) / c_{0}\left(X_{\alpha}\right)=X_{\alpha+1} \\
& : z \rightarrow\left[\left(g_{1}(z), g_{2}(z), g_{3}(z), \ldots\right)\right]
\end{aligned}
$$

where [.] denotes the equivalence class of the sequence in $l_{\infty}\left(X_{\alpha}\right) / c_{0}\left(X_{\alpha}\right)$. Then $G(z)$ is an analytic function $U_{1} \rightarrow X_{\alpha+1}$, and it is easy to see that $\left(z I-T_{\alpha+1}\right) G(z)$ is identically equal to the constant function

$$
\left[\left(d_{1}, d_{2}, d_{3}, \ldots\right)\right] \in X_{\alpha+1} .
$$

Therefore, by Corollary $4.5, G=\left[\left(d_{1}, d_{2}, d_{3}, \ldots\right)\right]=0$, contradicting our original hypothesis that each $\left\|d_{i}\right\|=1$, hence

$$
\left\|\left[\left(d_{1}, d_{2}, d_{3}, \ldots\right)\right]\right\|_{l_{\infty}\left(X_{\alpha}\right) / c_{0}\left(X_{\alpha}\right)}=\lim \sup \left\|d_{i}\right\|_{X_{\alpha}}=1 \text {. }
$$

So in fact there is an $\varepsilon>0$ which is admissible for the ordinal $\alpha$.

Next, we prove the following:

THEOREM 4.7. There is an $\varepsilon>0$ which is admissible for all $\alpha \in \Omega$.

PROOF. For each $\alpha \in \Omega$ let $\varepsilon_{1}(\alpha)$ denote the supremum of all values $\varepsilon_{1}$ such that $\varepsilon_{1}$ is admissible for $\alpha$. This is strictly positive by Lemma 4.6 , and it is a decreasing function of $\alpha$ because for each $\beta>\alpha$, and each fixed $\varepsilon$, the linking map

$$
\tau_{\alpha \beta}: X_{\alpha} \rightarrow X_{\beta}
$$

defines an isometry

$$
\chi_{\alpha \beta}:\left(V_{\alpha}^{(1)},\|\cdot\|_{\alpha}^{(\varepsilon)}\right) \rightarrow\left(V_{\beta}^{(1)},\|\cdot\|_{\beta}^{(\varepsilon)}\right)
$$

and

$$
\chi_{\alpha \beta}\left(Z_{\alpha}^{(1)}\right) \subset Z_{\beta}^{(1)} .
$$

Therefore there is a norm 1 map $\sigma_{\alpha \beta}$ induced by $\chi_{\alpha \beta}$,

$$
\sigma_{\alpha \beta}: Y_{\alpha}^{(1)}=V_{\alpha}^{(1)} / Z_{\alpha}^{(1)} \rightarrow V_{\beta}^{(1)} / Z_{\beta}^{(1)}=Y_{\beta}^{(1)}
$$

and if for all $x_{\beta} \in X_{\beta}$ we have $\left\|\Psi\left(x_{\beta}\right)\right\| \geq\left\|x_{\beta}\right\|$ then for all $x_{\alpha} \in X_{\alpha}$ we have

$$
\left\|\Psi_{\alpha}^{(1)}\left(x_{\alpha}\right)\right\| \geq\left\|\sigma_{\alpha \beta} \circ \Psi_{\alpha}^{(1)}\left(x_{\alpha}\right)\right\|=\left\|\Psi_{\beta}^{(1)}\left(\tau_{\alpha \beta}\left(x_{\alpha}\right)\right)\right\| \geq\left\|\tau_{\alpha \beta}\left(x_{\alpha}\right)\right\|=\left\|x_{\alpha}\right\| .
$$

We claim that the infimum $\inf _{\alpha \in \Omega}\left(\varepsilon_{1}(\alpha)\right)$ is strictly positive; for if for some ordinals $\alpha_{i} \in \Omega$ we have $\varepsilon_{1}\left(\alpha_{i}\right) \stackrel{i \rightarrow \infty}{\longrightarrow} 0$, then $\varepsilon_{1}\left(\bigcup_{1}^{\infty} \alpha_{i}\right)=0$, contradicting Lemma 4.6. Thus Theorem 4.7 is proved. We adopt a similar style to prove

THEOREM 4.8. If the sequence $\left(\varepsilon_{i}\right)_{i=1}^{n}$ is admissible for all $\alpha \in \Omega$ then for all $0<\delta<1$ there is an $\varepsilon_{n+1}>0$ such that the sequence $\delta \varepsilon_{1}, \delta \varepsilon_{2}, \delta \varepsilon_{3}, \ldots, \delta \varepsilon_{n}$, $\varepsilon_{n+1}$ is admissible for all $\alpha \in \Omega$.

The proof of Theorem 4.8 is quite similar to that of Theorem 4.7, and we begin with an analog of Lemma 4.6. 
LEMMA 4.9. Given that $\left(\varepsilon_{i}\right)_{i=1}^{n}$ is admissible for all $\alpha \in \Omega$, and given $\delta \in(0,1)$, the following is true.

For each $\beta \in \Omega$, there is an $\varepsilon_{n+1}$ such that the sequence $\delta \varepsilon_{1}, \delta \varepsilon_{2}, \delta \varepsilon_{3}, \ldots, \delta \varepsilon_{n}$, $\varepsilon_{n+1}$ is admissible for $\beta$. Let us write $\boldsymbol{\varepsilon}^{\prime}$ for this sequence.

PROOF. In view of (4.1.1) it is sufficient that we show that there is an $\varepsilon_{n+1}>0$ such that for all $x \in X_{\beta}$,

$$
\|\Psi(x)\|_{\beta}^{\varepsilon^{\prime}} \geq\|x\|_{X_{\beta}},
$$

where the norm in $V_{\beta}^{(n+1)}$ of the equivalence class of the constant function $x$ is

$$
\begin{aligned}
& \inf \left\{\|c\|_{X_{\beta}}+\sum_{i=1}^{n+1}\left\|f_{i}\right\|_{i}: c \in X_{\beta}, f_{i} \in V_{\beta}^{(i)}\right. \text {, and for some } \\
& g \in V_{\beta}^{(n+1)} \text { we have for all } z \in U_{n+1} \text {, } \\
& \left.c+\delta \cdot \sum_{1}^{n} \varepsilon_{i} f_{i}(z)+\varepsilon_{n+1} f_{n+1}(z)+\left(z I-T_{\beta}\right) \circ g(z)=x\right\} \text {. }
\end{aligned}
$$

If this is less than $\|x\|_{X_{\beta}}$ for a particular $x$ and $\varepsilon_{n+1}$, then for a suitable choice of $f_{i}$ 's, and a suitable choice of $c$, we have

$$
\begin{gathered}
c+\delta \cdot \sum_{1}^{n} \varepsilon_{i} f_{i}+\varepsilon_{n+1} f_{n+1}+(z I-T) \circ g=x, \\
\|c\|+\sum_{i=1}^{n+1}\left\|f_{i}\right\|_{\beta}^{(i)}<\|x\| .
\end{gathered}
$$

Writing $d=x-c$, we have

$$
\begin{gathered}
\delta \cdot \sum_{1}^{n} \varepsilon_{i} f_{i}+\varepsilon_{n+1} f_{n+1}+\left(z I-T_{\beta}\right) \circ g=d, \\
\sum_{1}^{n+1}\left\|f_{i}\right\|_{\beta}^{(i)}<\|x\|-\|c\| \leq\|d\| .
\end{gathered}
$$

Suppose there is no $\varepsilon_{n+1}>0$ such that the sequence $\varepsilon^{\prime}$ is admissible for $\beta$. Then there are elements $\left(d_{i}\right)_{i=1}^{\infty} \subset X_{\beta}$, without loss of generality $\left\|d_{i}\right\|=1$, such that for each $j$,

$$
d_{j}=\delta \cdot \sum_{1}^{n} \varepsilon_{i} f_{i}^{(j)}+2^{-j} f_{n+1}^{(j)}+\left(z I-T_{\beta}\right) \circ g^{(j)}
$$

with

$$
\sum_{i=1}^{n+1}\left\|f_{i}^{(j)}\right\|_{\beta}^{(i)}<1 \quad \text { and } \quad g^{(j)} \in V_{\beta}^{(n+1)} .
$$

Let $W_{n+1}$ be any open set such that $W_{n+1} \subset U_{n+1}, \bar{W}_{n+1} \cap \sigma_{e}(T)=\varnothing$, and $\partial W_{n+1} \supset \partial U_{n+1}$.

Then as in the proof of Lemma 4.4, there is an $\eta>0$ such that

$$
\left\|\left(\lambda I-T_{\beta}\right) x\right\| \geq \eta\|x\|
$$


for all $x \in X_{\beta}, \lambda \in W_{n+1}$. Hence for all $\lambda \in W_{n+1}, j \in \mathbf{N}$,

$$
\begin{aligned}
\left\|g^{(j)}(\lambda)\right\| & \leq \frac{1}{\eta} \cdot\left\|\left(\lambda I-T_{\beta}\right) \circ g^{(j)}(\lambda)\right\| \\
& =\frac{1}{\eta} \cdot\left\|d_{i}-\delta \cdot \sum_{1}^{n} \varepsilon_{i} f_{i}^{(j)}(\lambda)-2^{-j} f_{n+1}^{(j)}(\lambda)\right\| \\
& \leq \frac{1}{\eta} \cdot\left(1+\sum_{1}^{n} \delta \varepsilon_{i}\left\|f_{i}^{(j)}\right\|_{\beta}^{(n+1)}+2^{-j}\left\|f_{n+1}^{(j)}\right\|_{\beta}^{(n+1)}\right) \\
& \leq \frac{1}{\eta} \cdot\left(1+\delta \varepsilon_{1} \cdot \sum_{1}^{n}\left\|f_{i}^{(j)}\right\|_{\beta}^{(i)}+2^{-j}\left\|f_{n+1}^{(j)}\right\|_{\beta}^{(n+1)}\right) \\
& \leq \frac{2}{\eta}
\end{aligned}
$$

since we are assuming each $\varepsilon_{i}<1, \delta<1$, and $\sum_{1}^{n+1}\left\|f_{i}\right\|_{\beta}^{(i)} \leq 1$.

So the $g^{(j)}$ 's are uniformly bounded on $W_{n+1}$, hence, since $\partial W_{n+1} \supset \partial U_{n+1}$, the $g^{(j)}$ 's are uniformly bounded throughout $U_{n+1}$ by the maximum modulus principle. Indeed, they are uniformly differentiable on compact sets in $U_{n+1}$.

Extracting a subsequence as necessary, we may assume that for each $i=1,2$, $\ldots, n$ the norm $\left\|f_{i}^{(j)}\right\|_{\beta}^{(i)}$ tends to a limit $\lambda_{i}$ as $j \rightarrow \infty$. Then since for each $j$,

$$
\sum_{i=1}^{n+1}\left\|f_{i}^{(j)}\right\|_{\beta}^{(i)}<1
$$

it is certainly true that

$$
\sum_{i=1}^{n} \lambda_{i} \leq 1 .
$$

For each $i=1,2, \ldots, n$ the functions $f_{i}^{(j)}$ are uniformly bounded on $U_{i}$ as $j$ runs from 1 to $\infty$, and are uniformly differentiable on compact sets in $U_{i}$. Therefore for each $i \leq n$ the function

$$
\begin{aligned}
F_{i} & : U_{i} \rightarrow l_{\infty}\left(X_{\beta}\right) / c_{0}\left(X_{\beta}\right)=X_{\beta+1} \\
& : z \rightarrow\left[\left(f_{i}^{(1)}(z), f_{i}^{(2)}(z), f_{i}^{(3)}(z), \ldots\right)\right]
\end{aligned}
$$

is analytic, hence is an element of $V_{\beta+1}^{(i)}$.

Moreover

$$
\begin{aligned}
\left\|F_{i}\right\|_{\beta+1}^{(i)} & =\sup _{z \in U_{i}}\left\|\left[\left(f_{i}^{(j)}(z)\right)_{j=1}^{\infty}\right]\right\|_{X_{\beta+1}} \\
& =\sup _{z} \limsup _{j \rightarrow \infty}\left\|f_{i}^{(j)}(z)\right\| \leq \limsup _{j \rightarrow \infty}\left\|f_{i}^{(j)}\right\|_{\beta}^{(i)}=\lambda_{i} .
\end{aligned}
$$

Let us further define

$$
\begin{aligned}
G & : U_{n+1} \rightarrow X_{\beta+1} \\
& : z \rightarrow\left[\left(g^{(1)}(z), g^{(2)}(z), g^{(3)}(z), \ldots\right)\right] .
\end{aligned}
$$

$G$ is also analytic on $l_{\infty}\left(X_{\beta}\right) / c_{0}\left(X_{\beta}\right)$, since the $g^{(j)}$ 's are locally uniformly differentiable. 
Moreover, since for each $z \in U_{n+1}, j \in \mathbf{N}$,

$$
d_{j}=\delta \cdot \sum \varepsilon_{i} f_{i}^{(j)}(z)+2^{-j} f_{n+1}^{(j)}(z)+\left(z I-T_{\beta}\right) \circ g^{(j)}(z)
$$

and since the sequence $\left(2^{-j} f_{n+1}^{(j)}(z)\right)_{j=1}^{\infty}$ is certainly a null sequence in $l_{\infty}\left(X_{\beta}\right)$, it follows that in $l_{\infty}\left(X_{\beta}\right) / c_{0}\left(X_{\beta}\right)$ we have identically

$$
\delta \cdot \sum_{1}^{n} \varepsilon_{i} F_{i}(z)+\left(z I-T_{\beta+1}\right) \circ G(z)=\left[\left(d_{1}, d_{2}, d_{3}, \ldots\right)\right]
$$

for all $z \in U_{n+1}$. By Lemma 4.4, $G$ extends analytically to that domain on which the function

$$
\left.\left[\left(d_{1}, d_{2}, d_{3}\right), \ldots\right)\right]-\delta \cdot \sum_{1}^{n} \varepsilon_{i} F_{i}
$$

is defined, which is precisely $U_{n}$ since the $U_{i}$ 's are nested.

Hence writing $\hat{G}$ for this extension, we have for all $z \in U_{n}$,

$$
\left[\left(d_{1}, d_{2}, d_{3}, \ldots\right)\right]=\delta \cdot \sum_{1}^{n} \varepsilon_{i} F_{i}(z)+\left(z I-T_{\beta+1}\right) \circ \hat{G}(z) .
$$

Now

$$
\left\|\left[\left(d_{1}, d_{2}, d_{3}, \ldots\right)\right]\right\|_{X_{\beta+1}}=\limsup \left\|d_{i}\right\|_{X_{\beta}}=1 .
$$

But if we impose on $V_{\beta+1}^{(n)}$ the norm $\|\cdot\|_{\beta+1}^{\left(\varepsilon_{i}\right)_{1}^{n}}$ determined by the finite sequence $\varepsilon_{1}, \ldots, \varepsilon_{n}$, we find that

$$
\begin{aligned}
& \left\|\Psi_{\beta+1}^{(n)}\left(\left[\left(d_{i}\right)_{i=1}^{\infty}\right]\right)\right\| \\
& \quad=\inf \left\{\|c\|+\sum_{1}^{n}\left\|h_{i}\right\|_{\beta+1}^{(i)}: c+\sum_{1}^{n} \varepsilon_{i} h_{i}+\left(z I-T_{\beta+1}\right) \circ g=\left[\left(d_{i}\right)_{i=1}^{\infty}\right] \text { on } U_{n}\right\} \\
& \quad \leq \sum_{1}^{n}\left\|\delta F_{i}\right\|_{\beta+1}^{(i)} \quad\left(\text { with } c=0, h_{i}=F_{i}, g=\hat{G}, \text { and using }(4.9 .3)\right) \\
& \quad \leq \delta \quad(\text { by }(4.9 .2) \text { and }(4.9 .1)) \\
& \quad<1 .
\end{aligned}
$$

But then $\Psi_{\beta+1}^{(n)}$ is not an isometry, which contradicts the fact that the sequence $\left(\varepsilon_{i}\right)_{i=1}^{n}$ is admissible for $\beta+1$. So in fact there is an $\varepsilon_{n+1}>0$ such that the sequence $\delta \varepsilon_{1}, \delta \varepsilon_{2}, \ldots, \delta \varepsilon_{n}, \varepsilon_{n+1}$ is admissible for $\beta$.

PROOF OF THEOREM 4.8. Given $\left(\varepsilon_{i}\right)_{i=1}^{n}$ admissible for all $\alpha \in \Omega$, and $\delta \in$ $(0,1)$, by Lemma 4.9 there is, for each ordinal $\beta$, an $\varepsilon_{n+1}$ depending on $\beta$ such that the sequence $\delta \varepsilon_{1}, \delta \varepsilon_{2}, \ldots, \delta \varepsilon_{n}, \varepsilon_{n+1}$ is admissible for $\beta$. Let us write $\varepsilon_{n+1}(\beta)$ for the supremum of all such values $\varepsilon_{n+1}$. As in the proof of Theorem 4.7, we find that $\varepsilon_{n+1}(\beta)$ is a decreasing function of $\beta$. It must have a positive infimum, however, because if $\beta_{i} \in \Omega(i \in \mathrm{N}), \varepsilon_{n+1}\left(\beta_{i}\right) \stackrel{i \rightarrow \infty}{\longrightarrow} 0$, then $\varepsilon_{n+1}\left(\bigcup_{1}^{\infty} \beta_{i}\right)=0$, a contradiction.

So there is an $\varepsilon_{n+1}>0$, which is less than $\varepsilon_{n+1}(\beta)$ for all $\beta$, hence the sequence $\delta \varepsilon_{1}, \delta \varepsilon_{2}, \delta \varepsilon_{3}, \ldots, \delta \varepsilon_{n}, \varepsilon_{n+1}$ is admissible for all $\beta$. This concludes the proof of Theorem 4.8 . 
As an immediate corollary of Theorems 4.7 and 4.8 we have

THEOREM 3.5. There is a decreasing sequence $\left(\varepsilon_{i}\right)_{i=1}^{\infty}$ of strictly positive constants, such that for every $n \in \mathbf{N}$ and $\alpha \in \Omega$, the sequence $\left(\varepsilon_{i}\right)_{i=1}^{n}$ is admissible for $\alpha$.

\section{Proof of the main result.}

THEOREM 5. Given the Banach space $X$ and operator $T \in L(X)$, there is an extension $Y$ of $X$ such that $T$ extends continuously to $Y$, and the spectrum of $T$ in $Y$ is precisely the approximate point spectrum of $T$ in $X$.

PROOF. Let $\left(\varepsilon_{i}\right)_{i=1}^{\infty}$ be a sequence of positive reals such that $\left(\varepsilon_{i}\right)_{i=1}^{n}$ is admissible for every $\alpha \in \Omega$.

Let $V$ be the vectorspace of all analytic functions into $X$ which are defined on a neighborhood of $\sigma_{\mathrm{ap}}(T)$. On $V$ we define a seminorm

$$
\begin{aligned}
& \|f\|=\inf \left\{\|d\|_{X}+\sum_{i=1}^{n}\left\|f_{i}\right\|_{1}^{(i)}: n \in \mathbf{N}, d \in X, f_{i} \in V_{1}^{(i)},\right. \\
& \text { and for some } g \in V_{1}^{(n)} \text { we have for all } \lambda \in U_{n}, \\
& \left.\qquad d+\sum_{i=1}^{n} \varepsilon_{i} f_{i}(\lambda)+(\lambda I-T) \circ g(\lambda)=f(\lambda)\right\} .
\end{aligned}
$$

We know that $\|\cdot\|$ is always finite because every $f$ defined on a neighborhood of $\sigma_{\text {ap }}(T)$ is defined on $U_{n}$ for large enough $n$. It is immediate from the definition of an admissible sequence that if for all $n$ we have $\varepsilon_{1}, \varepsilon_{2}, \ldots, \varepsilon_{n}$ is admissible for $\alpha=1$ $\left(X_{1}=X\right)$, then the norm of a constant function $c$ in $V$ will be precisely $\|c\|_{X}$. So $X$ is isometrically embedded in $V$ as the constant functions. Moreover, the map $T \in L(X)$ extends to a map $\hat{T} \in L(V)$, where

$$
\hat{T}(f)=T \circ f .
$$

Examining the definition of the norm on $V$, we find that $\|\hat{T}\|=\|T\|$. However, if $\mu \notin \sigma_{\mathrm{ap}}(T)$, then $\mu I-T$ has an inverse in $L(V)$, namely the map

$$
\begin{aligned}
R_{\mu} & : V \rightarrow V \\
& : f \rightarrow g
\end{aligned}
$$

where $g(\lambda)=(\mu-\lambda)^{-1} \cdot f(\lambda)$. We claim this map is continuous on $(V,\|\cdot\|)$. Fix an $m$ such that $\mu \notin \bar{U}_{m}$. If $f \in V$, and $\varepsilon>0$ is given, we can write

$$
f(\lambda)=d+\sum_{1}^{n} \varepsilon_{i} f_{i}(\lambda)+(\lambda I-T) \circ g(\lambda)
$$

on $U_{n}$, where

$$
\|d\|_{X}+\sum_{1}^{n}\left\|f_{i}\right\|_{1}^{(i)} \leq\|f\|_{V}+\varepsilon .
$$


Without loss of generality, $m \leq n$. Then for all $\lambda \in U_{n}$,

$$
\begin{aligned}
\left(R_{\mu}(f)\right)(\lambda)= & (\mu-\lambda)^{-1} \cdot\left(d+\sum_{1}^{n} \varepsilon_{i} f_{i}(\lambda)+(\lambda I-T) \circ g(\lambda)\right) \\
= & \left((\mu-\lambda)^{-1} \cdot\left(d+\sum_{1}^{m} \varepsilon_{i} f_{i}(\lambda)\right)\right) \\
& +\sum_{m+1}^{n} \varepsilon_{i} \cdot(\mu-\lambda)^{-1} f_{i}(\lambda)+(\lambda I-T) \circ\left((\mu-\lambda)^{-1} g(\lambda)\right) \\
= & \sum_{m}^{n} \varepsilon_{i} f_{i}^{\prime}(\lambda)+(\lambda I-T) \circ g^{\prime}(\lambda),
\end{aligned}
$$

where for $\lambda \in U_{n}$,

$$
g^{\prime}(\lambda)=(\mu-\lambda)^{-1} \cdot g(\lambda)
$$

for $\lambda \in U_{m}$,

$$
f_{m}^{\prime}(\lambda)=\varepsilon_{m}^{-1} \cdot(\mu-\lambda)^{-1} \cdot\left(d+\sum_{1}^{m} \varepsilon_{i} f_{i}(\lambda)\right)
$$

and for each $m<i \leq n$, and each $\lambda \in U_{i}$,

$$
f_{i}^{\prime}(\lambda)=(\mu-\lambda)^{-1} \cdot f_{i}(\lambda)
$$

So

$$
\begin{aligned}
\sum_{i=m}^{n}\left\|f_{i}^{\prime}\right\|_{1}^{(i)} & =\sum_{i=m}^{n} \sup _{\lambda \in U_{i}}\left\|f_{i}^{\prime}(\lambda)\right\| \\
& \leq\left(\sup _{\lambda \in U_{m}}\left|(\mu-\lambda)^{-1}\right|\right) \cdot\left\{\varepsilon_{m}^{-1} \cdot\left(\|d\|+\sum_{1}^{n} \varepsilon_{i}\left\|f_{i}\right\|_{i}^{(i)}\right)+\sum_{m+1}^{n}\left\|f_{i}\right\|_{1}^{(i)}\right\} \\
& \leq \frac{1}{\varepsilon_{m} \cdot d\left(\mu, U_{m}\right)} \cdot\left(\|f\|_{V}+\varepsilon\right) .
\end{aligned}
$$

Hence, $\left\|R_{\mu}\right\| \leq 1 /\left(\varepsilon_{m} \cdot d\left(\mu, U_{m}\right)\right)$. So $R_{\mu}$ is indeed continuous. Furthermore, $R_{\mu}$ is an inverse for $\mu I-\hat{T}$, because for all $f \in V$,

$$
\left(R_{\mu} \circ(\mu I-\hat{T})\right) f=\left((\mu I-\hat{T}) \circ R_{\mu}\right) f=g
$$

where

$$
g(\lambda)=(\mu-\lambda)^{-1}(\mu f(\lambda)-T \circ f(\lambda))
$$

so

$$
\begin{aligned}
g(\lambda)-f(\lambda) & =\frac{\mu f(\lambda)-T \circ f(\lambda)}{\mu-\lambda}-f(\lambda) \\
& =\frac{\lambda f(\lambda)-T \circ f(\lambda)}{\mu-\lambda}=(\lambda I-T) \circ \frac{f(\lambda)}{\mu-\lambda} .
\end{aligned}
$$

Hence $\|g-f\|_{V}=0$, so $R_{\mu}$ is indeed an inverse for $\mu I-T$ on $V$.

It remains only to consider the completion $Y$ of the quotient space $V / Z$, where $Z$ is the subspace of all functions in $V$ of norm zero, in order to have a genuine Banach space to which $\hat{T}, R_{\mu}$ extend in an obvious way, so that $Y$ is an extension of $X$ to which $T$ extends in a natural way and loses all but its approximate point spectrum. This proves the theorem. 
6. A counterexample concerning two elements of a Banach algebra. In this section we exhibit a commutative unital Banach algebra $L$ containing elements $g_{1}$ and $g_{2}$ such that

(1) Both $g_{1}$ and $g_{2}$ are norm increasing (see below for definition).

(2) However, in no extension $L^{\prime}$ of $L$ is it possible to remove the compact set $\frac{1}{2} D$ ( $D$ the closed unit disk in $\mathbf{C}$ ) from the residual spectrum of both $g_{1}$ and $g_{2}$.

$L$ is built up from a family of Banach algebras $L(N, k)$ which we proceed to describe.

DEFINITION 6.1.

(a) The essential spectrum of the element $x$ in a commutative unital Banach algebra $A$ over $\mathbf{C}$ is

$$
\left\{\lambda \in \mathbf{C}: \lambda 1_{A}-x \text { is a topological zero divisor }\right\} .
$$

(b) The residual spectrum of $x$ consists of all points of the spectrum which are not in the essential spectrum.

(c) The element $x$ is norm increasing if $\|a x\| \geq\|a\|$ for all $a \in A$.

Note that if $x$ is norm increasing then int $D$ ( $D$ the unit disk in $\mathbf{C}$ ) contains no point in the essential spectrum of $x$, and if we also know that $x$ is not invertible, then int $D$ is contained in the residual spectrum of $x$ (for the boundary of the spectrum is contained in the essential spectrum). It is therefore natural to hope that compact sets like $\frac{1}{2} D$ can be removed simultaneously from the spectra of a finite family of norm increasing elements; but this example will show that this is not true for more than one element at a time.

Given any $N>3$ and $k \in \mathbf{N}$, we shall exhibit an algebra $L(N, k)$ with the following properties.

(1) $L(N, k)$ is a commutative unital Banach algebra.

(2) $L$ contains two norm increasing elements $g_{1}$ and $g_{2}$, both of norm $L$.

(3) If $M$ is an extension of $L(N, k)$ containing inverses $g_{1}^{-1}$ and $g_{2}^{-1}$ to $g_{1}, g_{2}$, then

$$
\left\|g_{1}^{-k}\right\|+\left\|g_{2}^{-k}\right\| \geq(N-1) \cdot N^{k-1}
$$

CONSTRUCTION OF $L(N, k)$. Let $B=B\left(b_{0}, b_{1}, b_{2}, g_{1}, g_{2}\right)$ consist of the elements

$$
\begin{aligned}
g_{1}^{i} g_{2}^{j}, & \text { all } i \geq 0, j \geq 0, \\
b_{0} g_{1}^{i} g_{2}^{j}, & \text { all } i \geq 0, j \geq 0, i \wedge j<k, \\
b_{l} g_{1}^{i} g_{2}^{j}, & \text { all } i \geq 0, j \geq 0, l=1 \text { or } 2 .
\end{aligned}
$$

Put

$$
\begin{aligned}
& \left\|g_{1}^{i} g_{2}^{j}\right\|=N^{i+j}, \\
& \left\|b_{0} g_{1}^{i} g_{2}^{j}\right\|=(N-1) \cdot N^{\left(k-1+(i \vee j-k)_{+}\right)} \\
& \left\|b_{l} g_{1}^{i} g_{2}^{j}\right\|=N^{i+j} .
\end{aligned}
$$

Let $L(N, k)$ be the Banach space with basis $B$, consisting of formal sums

$$
X=\sum_{b \in B} \lambda_{b} \cdot b
$$

with norm

$$
\|X\|=\sum_{b \in B}\left|\lambda_{b}\right| \cdot\|b\|<\infty
$$


Define a commutative product in $L(N, k)$ by the relations

$$
\begin{aligned}
b_{i} b_{j} & =0, \quad i, j=0,1,2 ; \\
b_{0} g_{1}^{k} g_{2}^{k} & =b_{1} g_{1}^{k}+b_{2} g_{2}^{k} .
\end{aligned}
$$

With this product, we claim that $L(N, k)$ is a Banach algebra; the inequality $\|x y\| \leq\|x\| \cdot\|y\|$ holds true for the following reason.

Since $L(N, k)$ is isomorphic as a Banach space to $l_{1}$ with (not normalized but otherwise standard) basis $B$, it is sufficient to show that for each $b, b^{\prime} \in B,\left\|b b^{\prime}\right\| \leq$ $\|b\| \cdot\left\|b^{\prime}\right\| ;$ the interesting case to check is when

$$
\begin{aligned}
b & =b_{0} g_{1}^{i} g_{2}^{j} & & (\text { let us say } i<k), \\
b^{\prime} & =g_{1}^{s} g_{2}^{t} & & (i+s \geq k, j+t \geq k) .
\end{aligned}
$$

It turns out that in this case

$$
\begin{aligned}
\left\|b b^{\prime}\right\| & =2 \cdot N^{i+j+s+t-k} \\
\|b\| \cdot\left\|b^{\prime}\right\| & \geq(N-1) \cdot N^{i \vee j-1+s+t} \\
& \geq\|b\| \cdot\left\|b^{\prime}\right\|,
\end{aligned}
$$

since $N \geq 3$ and $i<k$. So $L(N, k)$ is indeed a Banach algebra. We wish to show that $g_{1}$ and $g_{2}$ are norm increasing. Let us do this for $g_{2}$. By considering the effect of multiplication by $g_{2}$ on the basis elements, it suffices to prove the following inequalities.

$$
\begin{aligned}
B & =\left\|z_{0} b_{0} g_{1}^{m} g_{2}^{k-1}+z_{2} b_{2} g_{1}^{m-k} g_{2}^{k-1}\right\| \\
& \leq\left\|\left(z_{0} b_{0} g_{1}^{m} g_{2}^{k-1}+z_{2} b_{2} g_{1}^{m-k} g_{2}^{k-1}\right) g_{2}\right\|=A
\end{aligned}
$$

$\left(z_{0}, z_{2} \in \mathbf{C}, m \geq k\right)$. Now,

$$
\begin{aligned}
A & =\left\|z_{0} b_{1} g_{1}^{m}+z_{0} b_{2} g_{1}^{m-k} g_{2}^{k}+z_{2} b_{2} g_{1}^{m-k} g_{2}^{k}\right\| \\
& =\left|z_{0}\right| N^{m}+\left|z_{0}+z_{2}\right| N^{m}
\end{aligned}
$$

and

$$
B=\left|z_{0}\right|(N-1) N^{m-1}+\left|z_{2}\right| N^{m-1},
$$

thus

$$
A-B=\left|z_{0}\right| N^{m-1}+\left|z_{0}+z_{2}\right| N^{m}-\left|z_{2}\right| N^{m-1} \geq 0 .
$$

Therefore $g_{1}$ and $g_{2}$ are norm increasing.

But suppose $M$ is a superalgebra containing $L$, such that $g_{1}^{-1}, g_{2}^{-1} \in M$. Since $b_{0} g_{1}^{k} g_{2}^{k}=b_{1} g_{1}^{k}+b_{2} g_{2}^{k}$, we have

$$
b_{0}=b_{1} g_{2}^{-k}+b_{2} g_{1}^{-k}
$$

Therefore

$$
(N-1) N^{k-1}=\left\|b_{0}\right\| \leq\left\|g_{2}^{-k}\right\|+\left\|g_{1}^{-k}\right\| .
$$

Let us choose a fixed value of $N$, say $N=4$. Then we have generating sets $B_{k}=B_{k}\left(b_{0}, b_{1}, b_{2}, g_{1}, g_{2}\right)$. We aim to get a space containing norm increasing vectors $g_{1}$ and $g_{2}$, such that $(*)$ holds for every $k$ in any extension containing inverses $g_{1}^{-1}$, $g_{2}^{-1}$. 
To do this, we identify all the elements $g_{1} \in B_{k}$, and identify all the elements $g_{1} \in B_{k}$, and identify all the elements $g_{2} \in B_{k}$, but we label the various elements $b_{0}^{(k)}, b_{1}^{(k)}, b_{2}^{(k)} \in B_{k}$.

Thus we obtain a new generating set

$$
B_{\infty}=B_{\infty}\left(g_{1}, g_{2}, b_{0}^{(k)}, b_{1}^{(k)}, b_{2}^{(k)}(k=1,2, \ldots)\right) .
$$

We put norms on the various elements of $B_{\infty}$ exactly as before; and we define a new Banach space $L$ of formal sums

$$
X=\sum_{b \in B_{\infty}} \lambda_{b} b, \quad \lambda_{b} \in \mathbf{C}
$$

such that

$$
\|X\|=\sum_{b \in B \infty}\left|\lambda_{b}\right|<\infty .
$$

We define a commutative product on $L$ subject, as before, to the relations

$$
\begin{aligned}
b_{i}^{(k)} b_{j}^{(k)} & =0 \quad(k \in \mathbf{N}, i, j=0,1,2), \\
b_{0}^{(k)} g_{1}^{k} g_{2}^{k} & =b_{1}^{(k)} g_{1}^{k}+b_{2}^{(k)} g_{2}^{k}
\end{aligned}
$$

and we add on the new relations

$$
b_{i}^{(k)} b_{j}^{(l)}=0 \quad(k \neq l) .
$$

With this product $L$ is a Banach algebra, and $g_{1}, g_{2}$ are norm increasing vectors. But if $M$ is an extension of $L$ containing vectors $g_{1}^{-1}, g_{2}^{-2}$, then since

$$
b_{0}^{(k)}=b_{1}^{(k)} g_{2}^{-k}+b_{2}^{(k)} g_{1}^{-k}
$$

we have

$$
(N-1) N^{k-1}=\left\|b_{0}^{(k)}\right\| \leq\left\|g_{1}^{-k}\right\|+\left\|g_{2}^{-k}\right\|
$$

for every $k \in \mathbf{N}$.

Therefore, in particular, the radius of convergence of the power series

$$
\sum_{k=0}^{\infty} \lambda^{k} g_{i}^{-k}
$$

must be less than or equal to $1 / N$ either for $i=1$ or for $i=2$. Therefore in no such extension can we eliminate the compact set $\frac{1}{2} B(\mathbf{C})$ from the residual spectrum of $g_{1}$ and the residual spectrum of $g_{2}$; for if we could, then the radii of convergence would be at least $1 / 2$.

This completes our counterexample.

Note that, as a corollary, we cannot eliminate the "joint residual spectrum" of $g_{1}$ and $g_{2}$, if "joint residual spectrum" is defined in a sensible way. Recalling that the joint spectrum of $g_{1}$ and $g_{2}$ is by definition

$$
\left\{(\lambda, \mu) \in \mathbf{C} \times \mathbf{C}: \text { there are no } a_{1}, a_{2} \in L \text { with }\left(\lambda 1-g_{1}\right) a_{1}+\left(\mu 1-g_{2}\right) a_{2}=1\right\},
$$

we see that the appropriate definitions are as follows:

DEFINITION. The joint essential spectrum of elements $x$ and $y$ in a commutative unital Banach algebra $A$ is the set

$$
\left\{(\lambda, \mu) \in \mathbf{C} \times \mathbf{C}: \lambda 1-g_{1} \text { and } \mu 1-g_{2} \text { are joint topological zero divisors }\right\} .
$$


The joint residual spectrum is the collection of all elements in the joint spectrum which are not in the joint essential spectrum.

From this it is immediate that for each $\lambda$ in $\mathbf{C}$, there is a point $(\lambda, \mu)$ in the joint spectrum (respectively, joint essential spectrum) of $g_{1}$ and $g_{2}$ if and only if $\lambda$ is in the spectrum (respectively, the essential spectrum) of $g_{1}$. Hence we have the following corollary.

COROLLARY. There is no extension of $L$ in which the joint residual spectrum of $g_{1}$ and $g_{2}$ is eliminated. For eliminating the joint residual spectrum implies eliminating the residual spectra of both individual elements.

In [5], Bollobás exhibits a counterexample to a conjecture of Arens [4], consisting of a commutative unital Banach algebra containing norm increasing elements $g_{1}$, $g_{2}$, such that no extension can contain inverses $g_{1}^{-1}, g_{2}^{-2}$, with

$$
\left\|g_{i}^{-1}\right\| \leq 1 \quad(i=1,2) .
$$

His counterexample is isomorphic to my $L(N, 1)$. My own counterexample is obtained by sticking together countably many algebras $L(N, k)$ so as to obtain a further result: that one cannot find an inverse producing extension which eliminates $\frac{1}{2} B(\mathbf{C})$ from the residual spectra of both $g_{1}$ and $g_{2}$, no matter how large the norms of the inverses are allowed to be.

\section{REFERENCES}

1. C. J. Read, Inverse producing extension of a Banach algebra which eliminates the residual spectrum of one element, Trans. Amer. Math. Soc. 286 (1984), 715-725.

2. B. Bollobás, Adjoining inverses to commutative Banach algebras, Algebras in Analysis (J. H. Williamson, ed.), Academic Press, New York, 1975, pp. 256-257.

3. G. E. Shilov, On normed rings with one generator, Mat. Sb. $21(63)(1947), 25-46$.

4. Richard Arens, Linear topological division algebras, Trans. Amer. Math. Soc. 53 (1947), 623630.

5. B. Bollobás, Adjoining inverses to Banach algebras, Trans. Amer. Math. Soc. 181 (1973), 165-174.

6. __ Best possibie bounds to the norms of inverses adjoined to normed algebras, Studia Math. 51 (1974), 87-96.

7. C. J. Read, Extending an operator from a Hilbert space to a larger Hilbert space, so as to reduce its spectrum, Israel J. Math. (to appear).

Department of PuRE MAThematics and Mathematical Statistics, Cambridge UNIVERSITY, CAMBRIDGE, ENGLAND 